\title{
On the existence of a scattering pre-peak in the mono-ols and diols
}

\author{
Martina Požar ${ }^{1,2}$ and Aurélien Perera ${ }^{1}$ \\ ${ }^{1}$ Laboratoire de Physique Théorique de la Matière Condensée (UMR CNRS \\ 7600), Université Pierre et Marie Curie, 4 Place Jussieu, F75252, Paris cedex 05, \\ France. \\ ${ }^{2}$ Department of Physics, Faculty of Sciences, University of Split, Ruđera \\ Boškovića 33, 21000, Split, Croatia.
}

\begin{abstract}
We report a computer simulation study of four 1,n-diols (1,2-ethanediol to 1,5-pentanediol). It is found that increasing the alkyl chain length increases chain-like clusters, and correspondingly the structure factor prepeak, just like for mono-ols. However, our calculated Xray intensities show that the pre-peak tends to diminish to a shoulder, in contrast with mono-ols where the pre-peak becomes more apparent with increasing alkyl chain. We attribute this contrasting finding to the fact that the alkyl chain is constrained between the two hydroxyl groups in linear diols, while they are free in linear mono-ols.
\end{abstract}

\section{Introduction}

Associated liquids differ from simple liquids since these latter contain only free particles, while the former contain in addition various "living" clusters of associated particles[1,2]. Perhaps the simplest way to represent this difference, would be to compute, for both systems, the cluster probability distribution as a function cluster size. Even though such distribution is inherently biased by the various criteria to decide how a given particle belongs to a cluster [3, 4], the two types of distribution differ by the fact that, for simple liquids this distribution is a monotonously decaying function[3, 4], while for associating liquids one expects 
a peak at some cluster size corresponding to typical representative of associated particles[5, 6]. Indeed, in a simple liquid, the probability of finding a monomer is always greater than a dimer, which is larger than for a trimer, and so forth. Therefore the cluster distribution is a monotonously decaying curve. Typical examples are hard sphere or Lennard-Jones liquids for the simple liquids [7]. For associating liquids, the existence of these living clusters should favour some characteristic size over isolated monomers, hence producing a non-monotonic cluster distribution. Typical examples for such associating liquids are linear alcohols, namely mono-ols[5,6]. A notable exception is water, for which the cluster distribution is found to be monotonous [8]. The rationalisation of this behaviour is that water has a tetrahedral coordination, which makes the probability of large clusters always smaller than that of smaller clusters[5, 6, 8]. The situation is entirely different for linear mono-ols, which form chain-like clusters, which decreases the monomer probability compared to that of some mean labile chain. Since these associated liquids contain both free monomers and chain-like associated monomers, it is tempting to consider them as a pseudo-mixture of two species: a mixture of monomers and labile clusters. This type of consideration has been previously considered only for water, for which the existence of two types of liquids has been a paradigm since the early works of Frank [9], and is exacerbated by the experimental evidence of two forms of high and low density amorphous ice (HDA and LDA) [10], and the recent controversies raised by the search for a putative liquidliquid phase separation $[11,12,13]$. It is quite intriguing that this mixture idea has been used for water, which has a monotonous cluster distribution, but not for linear mono-ols, which do show a singularity in the cluster distribution.

From the experimental side, radiation scattering experiments on alcohols, and in particular mono-ols, reveal a scattering pre-peak $[14,15,16,17,18,19]$. This pre-peak is absent from simple liquids such as the Lennard-Jones liquids and weakly polar liquids[20]. Therefore, the existence of a radiation pre-peak is one possible signature of particle association. For mono-ols, the pre-peak has been related to the existence of clusters [21]. Again, such pre-peak is absent for liquid water [22], signaling the intriguing peculiarity of this liquid[23]. Scattering prepeaks have been recently discussed in room temperature ionic liquids, in relation to the association of charged groups and their segregation from the neutral atomic groups $[24,25,26]$.

These two approaches to detect associating liquids can be profitably used for the case of linear diols. Indeed, in the case of linear mono-ols, the hydroxyl groups can associate freely in chain patterns, while the oily tails are randomly distributed. In diols, however, these tails are constrained by the second hydroxyl group attached at the other end. In such case, the length of the oily tail should play an interesting role in the association of the end hydroxyl groups. In turn, such a constraint should influence the clustering properties, and it would be interesting 
to examine how much these differ from associated liquids such as mono-ols and water. This study is also interesting in an attempt to classify various two-liquids.

In the present study we examine the structural properties of 1,2-ethanediol (ethylene glycol) and 1,4-butanediol, in comparison with methanol and ethanol, respectively, as well as 1,3-propanediol and 1,5-pentanediol, by using molecular dynamics simulation techniques. Ethylene glycol has been previously studied through computer simulations by several authors [27, 28, 29, 30]. These studies confirm that computer simulations of these liquids are robust enough when compared to experiments on real systems. The focus of the present paper differs from that of the previous studied principally because it is centered around the cluster and two-liquids structures, in relation to detecting these labile clusters through cluster and radiation scattering (Xray) analysis, conducted with computer simulation techniques. The main result of our study is that the presence of the oily chain between the hydroxyl groups hinders their chain association, when compared to the role of the free alkyl chains of mono-ols. In particular, we predict that the Xray scattering pre-peak of linear diols tends to vanish with increasing chain lengths, by merging with the main peak. This is in contrast with mono-ols where this tendency is exactly the opposite: the pre-peak and mean peak become more separated with increasing alcohol chain length[21]. This is even more surprizing, since we equally find evidence of chain-like association of the hydroxyl groups in both type of systems. The first evidence comes from the probability of chain-like clusters which increases with chain length, just like for mono-ols. The second evidence comes from the atomic structure factors of the hydroxyl groups, which equally show the increase of the pre-peak with alkyl chain length. The reason for this contrasting behaviour comes precisely from the fact that the methyl groups are constrained between the two hydroxyl groups, hence they contribute very differently to scattering intensities, than the mono-ols.

\section{Simulation details}

As in our previous computer simulation studies, we used the Gromacs program package [31]. We chose the TraPPe (Transferable Potential for Phase Equilibria)[32] for diols, while neat mono-ols were modeled with both TraPPe [33] and OPLS (Optimized Potentials for Liquid Simulations) [34] force fields for the sake of comparison. Previous works [27, 28, 29, 30] mostly used OPLS or modified OPLS force fields. In what structural properties of alcohols are concerned, the differences are minor, as shown below. It should be noted that the all present simulations allow for flexibility of the alkyl chains. However, this flexibility is limited, as was previously noted by other authors[27]. Table I summarises all the non-bonded force field parameters. For the diols, we use the notation M1 and M2, 
respectively, for the methyl/methylene sites closer to the hydroxyl group, and the next one down the alkyl chain.

The initial configurations were generated with Packmol[35] from the appropriate pdb files. All system sizes were chosen for $\mathrm{N}=1000$ molecules, which was found to be sufficient to ensure proper asymptotic decay of the various site-site pair correlation functions. The systems were simulating the ambient condition liquids, in the isobaric-isothermal (constant $\mathrm{NpT}$ ) ensemble. The temperature of $\mathrm{T}=300 \mathrm{~K}$ and pressure of $\mathrm{p}=1$ bar were kept constant with the v-rescale thermostat[36] and Parrinello-Rahman barostat[37].

The simulation protocol was the same for all alcohols. After assembling the initial configurations, the system energy was minimized, followed by equilibrations in the NpT ensemble for a total of $2 \mathrm{~ns}$. The subsequent production runs lasted $2 \mathrm{~ns}$ and yielded at least 1500 configurations for each alcohol.

The clustering of the all the atomic sites, and in particular the hydroxyl groups were computed. The cluster is defined as the group of particles where each particle has at least one connection with the neighbor particles. The connectivity criteria can be geometrical constraints, or for example the Hills energetic criteria where particles are consider to be connected if their attractive interaction energy is higher then their relative kinetic energy[38]. In this work, we use the Stillinger distance criteria [4] where the cutoff distance is defined by the first minima of the site-site pair distribution function. This way, the bonding between particles are indirectly related to their interactions as refleted by their pair distribution function. The cluster size distributions are calculated for the clustering of the like-like sites, using several different statistical approaches. The cluster size probability function is evaluated as:

$$
s_{n}=\frac{\sum_{k=1}^{N_{c}} s(n, k)}{\sum_{k=1}^{N_{c}} \sum_{j=1}^{N_{m o l}} s(j, k)}
$$

where $s_{n}$ is the probability for the cluster formed of $n$ sites, $s(k, n)$ represents the number of clusters of the size $n$ in the configuration $k$. Varying the contact distance between neighbouring atoms that are part of a cluster distance around the first minima, shows a relative robustness in the resulting cluster distributions [5, 6]. The cutoff distances defined in this work are $r_{c}=3.7 \AA$ between oxygen atoms, $r_{c}=$ $4.5 \AA$ between the M1 and M2 pseudo atoms (see Table I). The cluster distribution features are quite robust to this choice, except in the case of ethanediol, which we discuss later below. Cluster size distributions were calculated with the Gromacs module g_clustsize.

The coordination number between atoms $i$ and $j$ is defined as:

$$
n_{i j}(r)=4 \pi \frac{N_{i}}{V} \int_{0}^{r} r^{2} g_{i j}(r) d r
$$


where $N_{i}$ is the number of atoms of types $i$ in the volume $V$.

The atom-atom structure factors are defined in relation to the Fourier transforms of the site-site pair correlation functions $g_{i j}(r)$

$$
S_{i j}(k)=\delta_{i j}+\rho \int d \vec{r}\left[g_{i j}(r)-1\right] \exp (\overrightarrow{i k} \cdot \vec{r})
$$

The radiation scattering experiments, in particular Xray scattering experiments such as SAXS and WAXS (small angle and wide angle Xray scattering) measure the scattering intensity, for which we use the Pings-Wasers expression [39], which conveniently allows to express this quantity in terms of the individual structure factors:

$$
I(k)=\sum_{i} f_{i}(k)^{2}+\rho \sum_{i, j} f_{i}(k) f_{j}(k) \tilde{h}_{i j}(k)
$$

where the sum runs over all type of atoms and the f-functions are the atomic form factors[40]. $\rho=N / V$ is the number of particles $N$ per volume $V$. The $\tilde{h}_{i j}(k)$ functions are the Fourier transforms of the $h_{i j}(r)=g_{i j}(r)-1$. One can rewrite this expression by using the definition of the structure factor in Eq.(2) as:

$$
I(k)=\sum_{i j} f_{i}(k) f_{j}(k) S_{i j}(k)
$$

The first term in Eq.(3) relates to the ideal contribution to the scattering in the absence of pair correlations (which is also equivalent to $\rho=0$ )

$$
I_{\text {ideal }}(k)=\sum_{i} f_{i}(k)^{2}
$$

We have calculated the Xray scattering intensities using the expressions above. We do not report here the neutron scattering intensities, which differ from the Xray data through the fact that the form factors $f_{i}$ are constants (independent of $k$ ) for neutron scattering, while they have a Gaussian-like shape for Xray scattering. One reason for not reporting this data is because the form factors vary according to whether the various atoms are deuterated or not, which offers too many combinations. The Xray data is independent of such constraints.

Since the force field models used here account for the methyl and methylene groups as a single united atom, it was necessary to find an appropriate form factor for the united atom representation. The procedure chosen here is quite simple: the central carbon-carbon pair correlations and carbon-hydrogen pair correlations are assumed to be the same to the united atom self pair correlations. This amounts to the approximation ( $C$ stands for carbon and $H$ for hydrogen)

$$
h_{C C}=h_{C H}=h_{M M}
$$


This is reasonable when there are no charge interactions, and when the sites of the united atom representation are close to each other, which is clearly the case for the carbon and hydrogens atoms associated to methyl and methyle group. If we plug in the approximation Eq.6 into Eq.4, it is quite easy to see that the form factor of the united atom becomes

$$
f_{M}(k)=f_{C}(k)+n f_{H}(k)
$$

where $n$ is the number of the hydrogen atoms in the methyl or methylene group.

\section{Results}

We will mostly show comparative results for ethanediol and methanol, and butanediol and ethanol. Results for propanediol and pentanediol are commented whenever necessary. However, we report the calculated intensities for all four diols.

\subsection{Snapshots}

Fig. 1 shows snapshots of the four alcohols. While chain-like patterns of the hydroxyl groups are more apparent in ethanol and butanediol, the case of methanol and ethanediol deserve some comments. From previous studies [41, 42, 43], it is known that methanol has chain-like associations. This is not so apparent in the snapshot as it is for the higher alcohols. The case of ethanediol is more interesting. One sees much less chains, and these tend to be shorter than in methanol. But, one also sees few chains aligned next to each other. This type of alignment overall destroys the single chain detection in the cluster algorithm. Although not shown here, propanediol and pentanediol show chain behaviour similar to butandiol, with a more pronounced chaining for pentanediol.

\subsection{Cluster distribution}

In order to confirm the visual analysis, we compare in the main panel of Fig. 2 the cluster probability distributions of the hydroxyl oxygen atoms, between the four neat liquids. It is seen that, while methanol and ethanol oxygens have a cluster peak about mean cluster size 5, which correspond to that observed in the corresponding snapshots, we note that the cluster structure of butanediol is very similar to that of the mono-ols, however with a smaller monomer probability. This implies that hydroxyl groups are less free in butandiol than in the mono-ols. This is somewhat counter-intuitive, since we expect that the constraint imposed by the alkyl chain would leave fewer hydroxyl groups free. Yet, we observe that there 
are more bound hydroxyl groups in diols than in the mono-ols. This is equally the case for propanediol and pentanediol (inset), which both show the cluster peak corresponding to the observed chaining of the hydroxyl groups. The case of ethanediol is strikingly different from the others. First, the specific peak is more of a shoulder than a peak, and second, it looks more like a cluster distribution of a simple liquid. This can be rationalized in terms of the constraint imposed by the alkyl chain: the number of free hydroxyl monomers is indeed larger than for the 3 other alcohols. The cutoff dependence (full versus dashed line) shows a larger dependence for ethanediol than for the other alcohols (not shown). The methyl/methylene groups of diols have a monotonously decaying cluster distribution, just like for neat mono-ols $[5,6]$. This is shown for butandiol in dashed lines in the inset. Since these groups go in pairs in each molecules, the monomer distribution is slightly lower than the dimers. This overall monotonous cluster distribution implies that, despite the constraint of being tied to the clustered hydroxyl groups, these methyl groups are essential randomly distributed.

\subsection{Pair correlation functions}

Fig3 shows the pair correlation functions for ethanediol (left panel) and methanol (right panel). The typical feature of a hydrogen bonded system are observed for the oxygen and hydrogen pair correlations: a first sharp peak, followed by depleted pair correlations of the nearest next neighbours. This depletion is due to the underlying charge ordering [44]. Indeed, hydrogen bond association is modeled by Coulomb interactions, and these impose the alternate distribution of plus and minus charges, which is called charge ordering[45, 46]. In a system constrained by the presence of neutral groups, charge ordering often takes the shape of chain-like pattern of the plus and minus charges, thus depleting their isotropic distribution. These typical features of increased first pair correlations, accompanied by a depressed pair correlation produces the pre-peak in the associated structure factor [44]. The carbon group pair correlations are very much LJ-like, as expected. The main difference between the ethanediol and methanol is in the height of the first peaks, and perhaps a more marked depletion of second neighbours. This is expected on the basis of the constrained versus free alkyl chains argument. For methanol, we have shown a comparison with the OPLS force field (in dashed lines) and it is clearly seen that the difference between the TraPPE and OPLS force fields is negligible, at least in what concerns structural properties.

Fig4 shows the pair correlation for butanediol(left) and ethanol(right). Features similar to those described above are seen again, as expected because of the similarities of these systems. We observe that the hydrogen bonding first peaks are higher than in the respective previous cases, while the depletion is not so much more marked. This is in support of the increased chaining pattern, and in align- 
ment with the observations of the previous sections. We note again that hydrogen bonding in butanediol is smaller than in ethanol. We equally note that the pair correlations between the methyl sites are in phase for ethanol, but dephased for butanediol. This is an important indication, which will serve us to explain the radiation scattering data below.

Fig. 5 shows the coordination number of the oxygen sites, which complements the information found in the snapshot and cluster distributions. It is found that the first peak and minimum of $g_{O O}(r)$ contributes to a marked inflexion of the coordination around 2 neighbours in average. This is fully consistent with the existence of linear clusters of the hydroxyl sites. This is less apparent for ethanediol, again in good agreement with the lesser presence of such clusters in this system.

The structural features for propanediol and pentanediol are very similar to butanediol, and are not reported here.

\subsection{Structure factors}

Fig.6 shows the various atom-atom structure factors of the pair correlations displayed in Fig.3. The presence of the pre-peak at $k_{P} \approx 1 \AA^{-1}$ is clearly noticeable. The main peak is given by the methyl site pair correlation at $k_{M} \approx 2 \pi / \sigma_{M}$, and depends on the methyl site diameter $\sigma_{M} \approx 3.5$. The pre-peak is more marked for the methanol than for the ethanediol pair correlations. This is consistent with the fact that the cluster structure of the latter system is less pronounced than that of the former. We equally note that the double peak structure of the pre-peak "plateau" like feature in $S_{O O}(k)$ is in fact due to the dual contributions of the $\mathrm{OH}$ peak at $k_{P} \approx 1 \AA^{-1}$ and the main peak at $k_{M} \approx 2 \pi / \sigma_{M}$. It is interesting to note that the pre-peak at $k_{P} \approx 1 \AA^{-1}$ is equally found in the recently reported total structure factor in neutron scattering experiments[47], and the whole shape is very similar to that we report here.

Fig.7 shows the structure factors for the butanediol and ethanol corresponding to the pair correlation shown in Fig.4. The pre-peak is much more pronounced than in the previous case, witnessing better cluster structures and confirming general trends deduced from previous analysis. We equally observe that the pair correlations between the methyl sites are out of phase for butanediol and in phase for ethanol.

The pre-peak feature for propanediol and pentanediol are quite similar to those reported above, with an increased pre-peak and decreasing main peak trend with increasing alkyl chain length. This is in line with the cluster plot (inset of Fig.2) and the visually observable chains in the snapshots for both these systems. Some of these structure factors are reported below in Fig.8. 


\subsection{Scattering intensity}

The Pings-Wasers Xray scattering intensity is calculated from the expression given in Eq.(3), with the atom-atom structure factors calculated and shown in the previous sub-section. Fig. 8 shows the SAXS data for all four diols, together with those calculated for the mono-ols, and experimental SAXS data from Ref.[21] for ethanol. In each panels, the main intensity $I(k)$ is reported in blue lines, and the ideal intensity $I_{\text {ideal }}(k)$ (Eq.5) in green lines (dashed lines for the mono-ols). It is seen that, despite the fact that the pre-peak structure of butanediol is better defined from that of ethanediol, the scattering intensity shows a less pronounced contribution for the former. This trend becomes more apparent for propanediol and pentanediol, for which only the main peak is apparent, and the pre-peak is just a shoulder. This is in variance with linear mono-ols, for which the pre-peak is more pronounced for longer chains[21]. We relate this finding with the fact that the carbon site contributions are i) more numerous for longer diols and ii) out of phase for the longer diols and distributed between $k_{P}$ and $k_{M}$. This contributes to enhance the contributions at $k_{M}$, while smearing that at $k_{P}$. This important finding remains to be confirmed experimentally as well.

It is interesting to note that the individual $S_{i j}(k)$ are not primary experimental observables, unlike $I(k)$. However, there exist procedures to rebuild the $S_{i j}(k)$ by isotope weighting techniques of the various atomic contributions. This holds only for neutron scattering data. In the case of Xray scattering, this usual procedure is to rebuild $\mathrm{I}(\mathrm{k})$ from the computed $S_{i j}(k)$. If the pre-feature is almost erased from the final $\mathrm{I}(\mathrm{k})$, one wonders which procedure would restore it in the individual $S_{i j}(k)$. It seems more likely that these contributions are likely to remain smeared, leading to discrepancies in the comparison between the simulated and re-computed $S_{i j}(k)$, which would be totally artificial.

\section{Discussion and Conclusion}

If the hydroxyl endgroup of any alcohol molecule is identified to a dipolar or magnetic "charge", then linear mono-ols are free "charges", while linear diols are constrained "charge" pairs, constrained by the intermediate alkyl chain. This analogy with magnetic systems, in particular with monopoles, which has attracted a recent renewed interest $[48,49]$, hints to the importance the constraint between the two hydroxyl groups can have. In this "molecular representation" of the magnetic problem, it is how the local order evolves with the constraint length, which becomes the appealing comparison factor. In this context, the association of these charges into chains bears a different meaning, according to the case when the charges are free or tied by the alkyl chains. What the present study shows 
is that, independently of the alkyl-chain tying constraints, increasing the chain length leads to better chain-like association of the hydroxyl "charges" both in mono-ols and diols. However, the presence of the constraint influences markedly the scattering function, by smearing the pre-peak and the main peak into a single feature. This means that experimental detection of the chain pre-peak in diols is rendered difficult or even impossible through radiation scattering experiments, as opposed to mono-ols. This means that, in the absence of an independent statistical description, such as computer simulations for example, it would be difficult to tell if specific forms of clusters can appear in some types of associating liquids, such as diols. This "invisibility" of a microscopic feature through a given experiment is an intriguing aspect of the present work.

This problem of the invisibility of a microscopic feature through radiation scattering experiments was equally met in our previous studies of aqueous mixtures of mono-ols (as well as other polar solutes), where strong pre-peak are observed in atom-atom structure factors obtained from simulations, while there seems to be no evidence of them in Xray or neutron scattering data[50]. The present system then provides one solution to this seemingly generic enigmatic finding between scattering and computer experiments, which concerns the problem of a direct experimental detection of the microscopic heterogeneity.

\section{Acknowledgments}

This work has been supported in parts by the Croatian Science Foundation for financial support under the project 4514 "Multi-scale description of meso-scale domain formation and destruction". M. Požar thanks the French Embassy in Croatia for financial support through "bourse du Gouvernement Français".

\section{References}

[1] A. Stradner, H. Sedgwick, F. Cardinaux, W. C. K. Poon, S. U. Egelhaaf and P. Schurtenberger, Nature 432, 492 (2004)

[2] A. J. Archer, N. B. Wilding, Phys. Rev. E 76, 031501 (2007)

[3] L. A. Pugnaloni and F. Vericat, J. Chem. Phys., 116, 1097 (2002).

[4] F. H. Stillinger, J. Chem. Phys., 38, 1486 (1963).

[5] A. Perera, F. Sokolic and L. Zoranic, Phys. Rev., E75, 060502-(R) (2007).

[6] L. Zoranic, F. Sokolic and A. Perera, J. Chem. Phys., 127, 024502 (2007). 
[7] H. Jonsson and H. C. Andersen, Phys. Rev. Lett., 60, 2295 (1988).

[8] A. Geiger, F. H. Stillinger and A. Rahman, J. Chem. Phys., 70, 4185 (1979).

[9] H.S. Frank and A.S. Quist, J. Chem. Phys. 34, 604 (1961)

[10] O. Mishima, L. D. Calvert and E. Whalley, Nature 314, 76-78 (1985)

[11] O. Mishima and H.E. Stanley, Nature 392, 164 ?168 (1998).

[12] P.H. Poole, F. Sciortino, U. Essmann, H. Stanley, Nature 360, 324 ?328 (1992).

[13] P. Gallo, K. Amann-Winkel, C. A. Angell, M. A. Anisimov, F. Caupin, C. Chakravarty, E. Lascaris, T. Loerting, A. Z. Panagiotopoulos, J. Russo, J. A. Sellberg, H. E. Stanley, H. Tanaka, C. Vega, L. Xu, and L. G. M. Pettersson, Chemical Reviews 116, 7463-7500 (2016).

[14] B. E. Warren, Phys. Rev. 44, 969 (1933).

[15] A. H. Narten and A. Habenschuss, J. Chem. Phys. 80, 3387 (1984)

[16] A. H. Narten and S. I. Sandler, J. Chem. Phys. 71, 2069 (1979)

[17] D. G. Montague, J. C. Dore and S. Cummings, Mol. Phys. 53, 1049 (1984)

[18] S. Sarkar and R. N. Joarder, J. Chem. Phys. 99, 2032 (1993)

[19] R. Böhmer, C. Gainaru and R. Richert, Phys. Rep. 545, 125 (2014)

[20] J.P. Hansen and I.R. McDonald, Theory of Simple Liquids (Academic, London, 1986)

[21] M. Tomšič, A. Jamnik, G. Fritz-Popovski, O. Glatter and L. Vlcek, J. Phys.Chem. B 111, 1738 (2007)

[22] T. Head-Gordon and H. Hura, Chem. Rev. 102, 2651 (2002)

[23] A. Perera, Mol. Phys. 109, 2433 (2011)

[24] A. Triolo, O. Russina, H-J Bleif and E. Di Cola, J. Phys. Chem. B111, 4641 (2007)

[25] H. V. R. Annapureddy, H. K. Kashyap, P. M. De Biase and C. J. Margulis, J. Phys. Chem. B114, 16838 (2010)

[26] Y. Wang, W. Jian, T. Yan and G. A. Voth, Acc. Chem. Res. 40, 1193 (2007) 
[27] L. Saiz, J. A. Padró, and E. Guàrdia, The Journal of Chemical Physics 114, 3187 (2001)

[28] A. V. Gubskaya and P. G. Kusalik, J. Phys. Chem. A 108, 7151-7164 (2004)

[29] O. Vital de Oliveira and L.C. Gomide Freitas, Journal of Molecular Structure: THEOCHEM 728, 179 (2005)

[30] A. Kaiser, O. Ismailova, A. Koskela, S.E. Huber, M. Ritter, B. Cosenza, W. Benger, R. Nazmutdinov, M. Probst, Journal of Molecular Liquids 189, 20 (2014)

[31] D. van der Spoel, E. Lindahl, B. Hess, G. Groenhof, A. E. Mark and H.J.C. Berendsen, J.Comp. Chem. 26, 1701 (2005)

[32] J.M. Stubbs, J.J. Potoff and J.I. Siepmann, J. Phys. Chem. B 108, 1759617605 (2004)

[33] B. Chen, J.J. Potoff and J.I. Siepmann, J. Phys. Chem. B 105, 3093-3104 (2001)

[34] W. L. Jorgensen, J. Tirado-Rives, J. Am. Chem. Soc. 1657 (1988)

[35] J. M. Martínez and L. Martínez, Journal of Computational Chemistry, 24(7):819-825, 2003; ibid L. Martínez, R. Andrade, E. G. Birgin, J. M. Martínez. Journal of Computational Chemistry 30, 2157 (2009)

[36] G. Bussi, D. Donadio and M. Parrinello, J. Chem. Phys. 126, 014101 (2007)

[37] M. Parrinello and A. Rahman, J. Appl. Phys. 52, 7182 (1981)

[38] Pugnaloni L. A. and Vericat F.(2002) J. Chem. Phys. 1161097 (2002)

[39] J. C. Pings and J. Waser, J. Chem. Phys. 48, 3016 (1968)

[40] International Tables for Crystallography, ed. E. Prince, International Union of Crystallography, 2006, vol. C.

[41] Y. Tanaka, N. Ohtomo, K. Arakawa, Bull. Chem. Soc. Jpn. 58270 (1985).

[42] T. Yamaguchi, K. Hidaka, A.K. Soper, Mol. Phys. 961159 (1999).

[43] J.-H. Guo, Y. Luo, A. Augustsson, S. Kashtanov, J.-E. Rubens- son, D. K. Shuh, H. Ågren, and J. Nordgren, Phys. Rev. Lett. 91, 157401 (2003)

[44] A. Perera and R. Mazighi, J. Chem. Phys., 143, 154502 (2015). 
[45] F. G. Edwards, J. E. Enderby and D. I. J. Page, Phys. C: Solid State Phys. 8 , 3483 (1975)

[46] A. Perera, Phys. Chem. Chem. Phys. DOI: 10.1039/c6cp07834f (2017)

[47] H. Abdelmoulahi, H. Ghalla, S. Nasr, M. Bahri and M.-C. Bellissent-Funel, J. Mol. Liq. 220, 527 (2016)

[48] S. Ladak, D. E. Read, G. K. Perkins, L. F. Cohen and W.R. Branford, Nature Physics 6, 359 (2010)

[49] E. Mengotti, L. J. Heyderman, A. F. Rodríguez, F. Nolting, R. V. Hügli and H.-B. Braun, Nature Physics 7, 68 (2011)

[50] A. Perera, Pure Appl. Chem. 88, 189 (2016) . 


\section{Table caption}

Table 1 - TraPPe and OPLS (only for methanol -data in parenthesis) force field parameters (non-bonded) for diols and mono-ols.

\begin{tabular}{|c|c|c|c|c|}
\hline Alcohol & Site & $\varepsilon / k_{B}[\mathrm{~K}]$ & $\sigma[\AA]$ & $q[e]$ \\
\hline \hline \multirow{4}{*}{ Methanol } & $\mathrm{CH}_{3}(\mathrm{M} 2)$ & $98.0(104.2)$ & $3.750(3.775)$ & $0.265(0.265)$ \\
\cline { 2 - 5 } & $O$ & $93.0(85.6)$ & $3.020(3.070)$ & $-0.700(-0.700)$ \\
\cline { 2 - 5 } & $H$ & $0.0(0.0)$ & $0.000(0.000)$ & $0.435(0.435$ \\
\hline \multirow{4}{*}{ Ethanol } & $\mathrm{CH}_{3}(\mathrm{M} 2)$ & 98.0 & 3.750 & 0.000 \\
\cline { 2 - 5 } & $\mathrm{CH}_{2}(\mathrm{M} 1)$ & 46.0 & 3.950 & 0.265 \\
\cline { 2 - 5 } & $O$ & 93.0 & 3.020 & -0.700 \\
\cline { 2 - 5 } & $H$ & 0.0 & 0.000 & 0.435 \\
\hline \multirow{3}{*}{ 1,4-ethanediol } & $\mathrm{CH}_{2}(\mathrm{M} 2)$ & 46.0 & 3.950 & 0.265 \\
\cline { 2 - 5 } & $O$ & 93.0 & 3.020 & -0.700 \\
\cline { 2 - 5 } & $H$ & 0.0 & 0.000 & 0.435 \\
\hline & $\mathrm{CH}_{3}(\mathrm{M} 2)$ & 46.0 & 3.950 & 0.000 \\
\cline { 2 - 5 } & $\mathrm{CH}_{2}(\mathrm{M} 1)$ & 46.0 & 3.950 & 0.265 \\
\cline { 2 - 5 } & $O$ & 93.0 & 3.020 & -0.700 \\
\cline { 2 - 5 } & $H$ & 0.0 & 0.000 & 0.435 \\
\hline
\end{tabular}




\section{Figure captions}

- Fig.1 - Snapshots: (a) ethanediol, (b) methanol, (c) butanediol, (d) ethanol. Oxygen atom is shown in red, hydrogen in white and methyl and methylene united atoms as semi-transparent tan.

- Fig. 2 - Cluster probability $P(s)$ versus cluster size $s$. The cutoff parameter is taken to be $r=3.6 \AA$, except for ethanediol for which $r=3 \AA$ and $r=2.9 \AA$ are reported in dashed and full red lines. Main panel shows a comparison between mono-ols and diols. The inset shows more diols distrubutions (full lines) and distribution of the methylene sites (M1 and M2) for butanediol).

- Fig.3 - Site-site pair correlation functions for ethanediol (left panel) and methanol (right panel).

- Fig.4 - Site-site pair correlation functions for butanediol (left panel) and ethanol (right panel)

- Fig.5 - Oxygen-oxygen coordination numbers. The $g_{O o}(r)$ function as shown in black.

- Fig.6 - Site-site structure factors corresponding to the pair correlations shown in Fig. 3 for ethanediol (left panel) and methanol (right panel).

- Fig.7 - Site-site structure factors corresponding to the pair correlations shown in Fig. 3 for butanediol (left panel) and ethanol (right panel)

- Fig.8 - Calculated Xray scattering intensity $I(k)$ for diols (in blue). The green curve is the ideal contribution (see text). The scattering curves for mono-ols (methanol and ethanol) are shown in dashed lines under those of ethanediol and butanediol. The experimental Xray data for ethanol[21] is shown in black line. The insets show typical atom-atom structure factors (see text) with a scaled $I(k)$ (in thick black line), with vertical lines indicating the position of the pre-peak and main peak. 
Figure 1

ACCEPTED MANUSCRIPT

a)
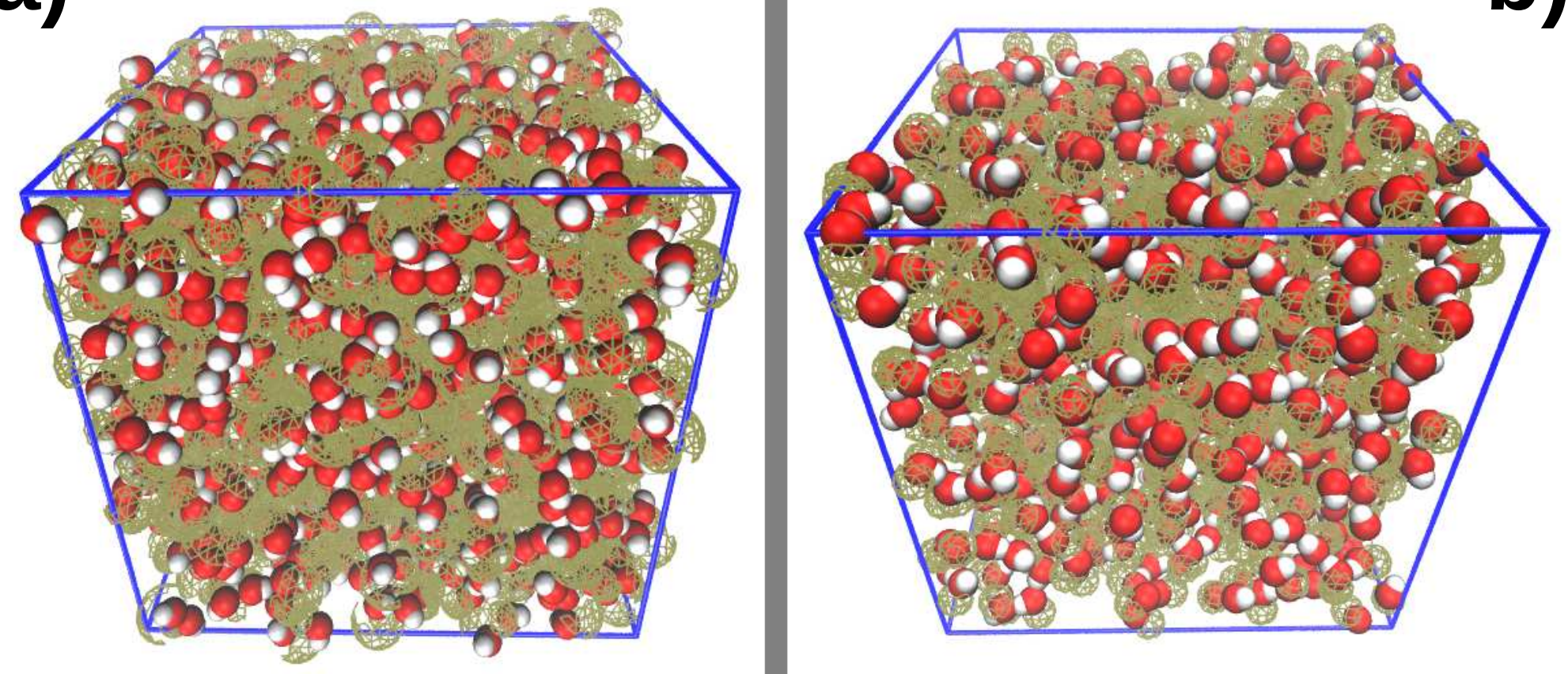

c)
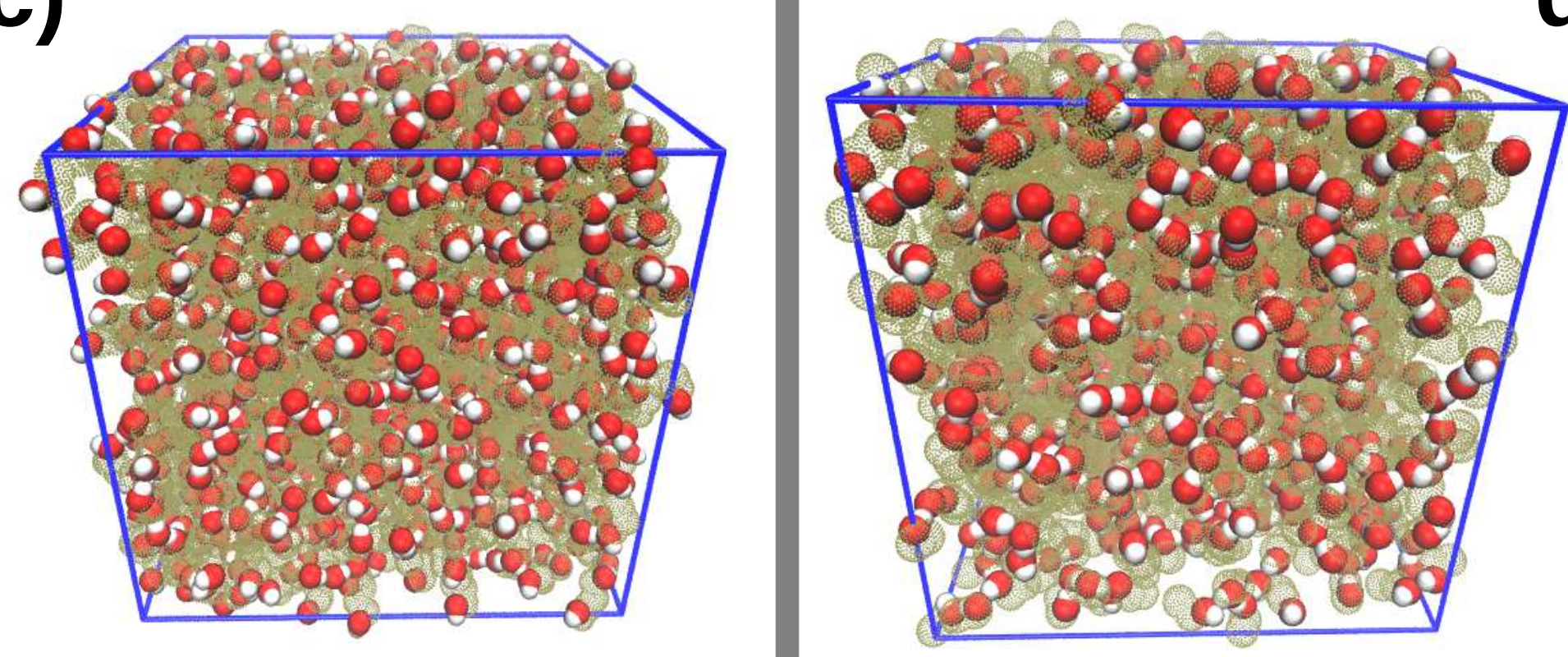

b)

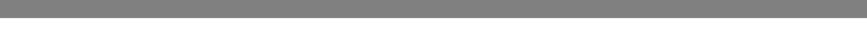

\section{d)}




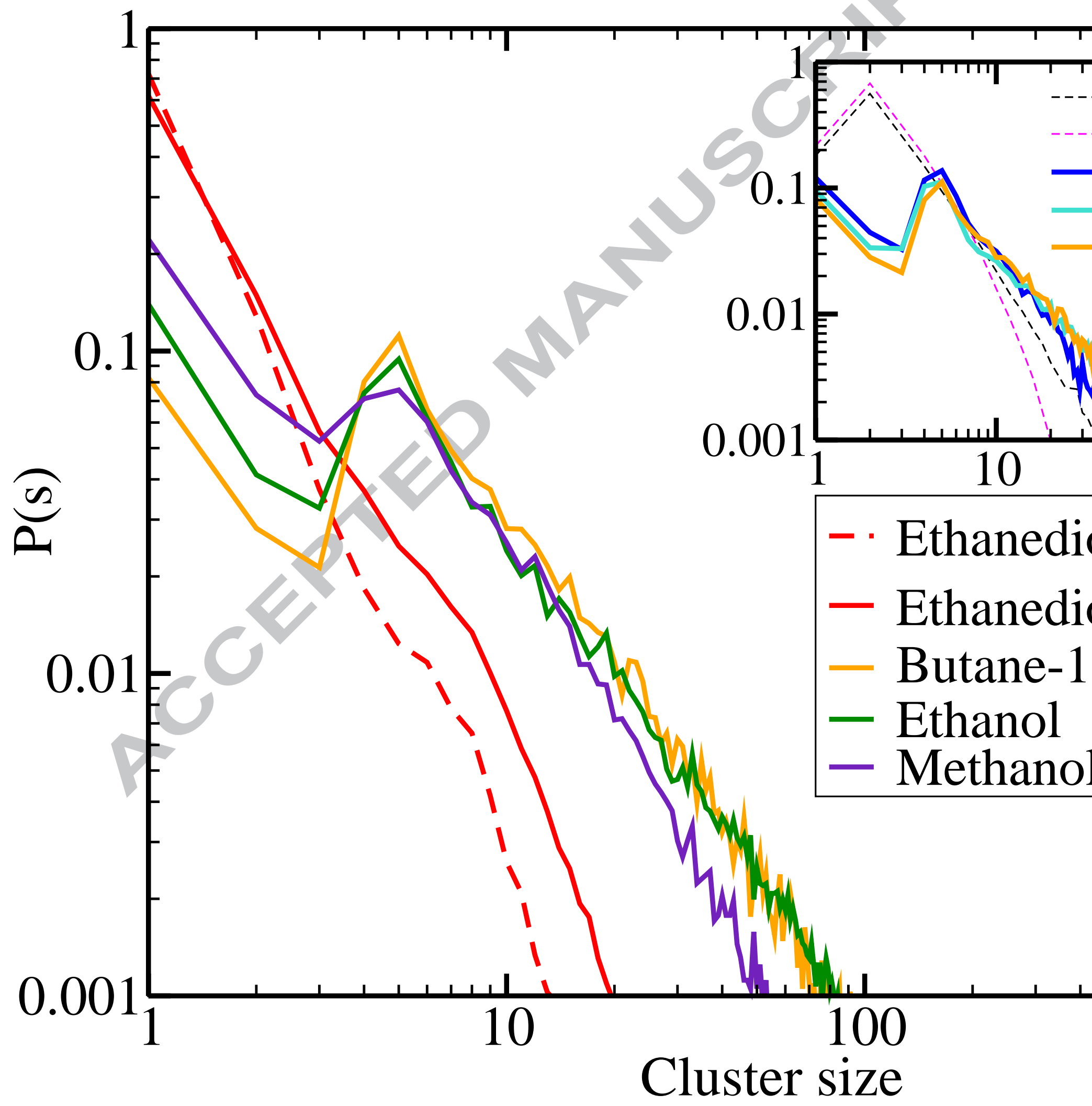




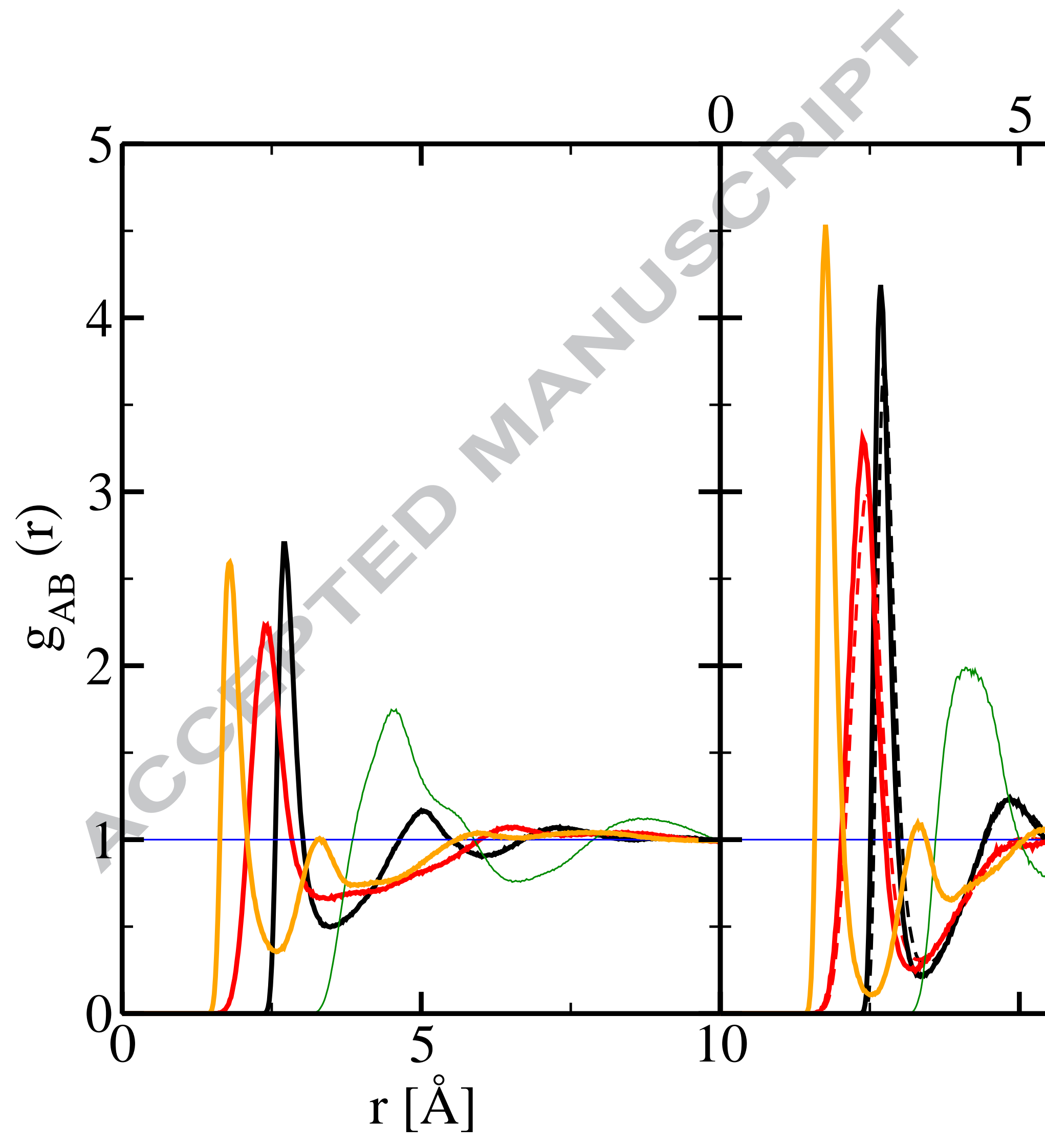

Figure 3 


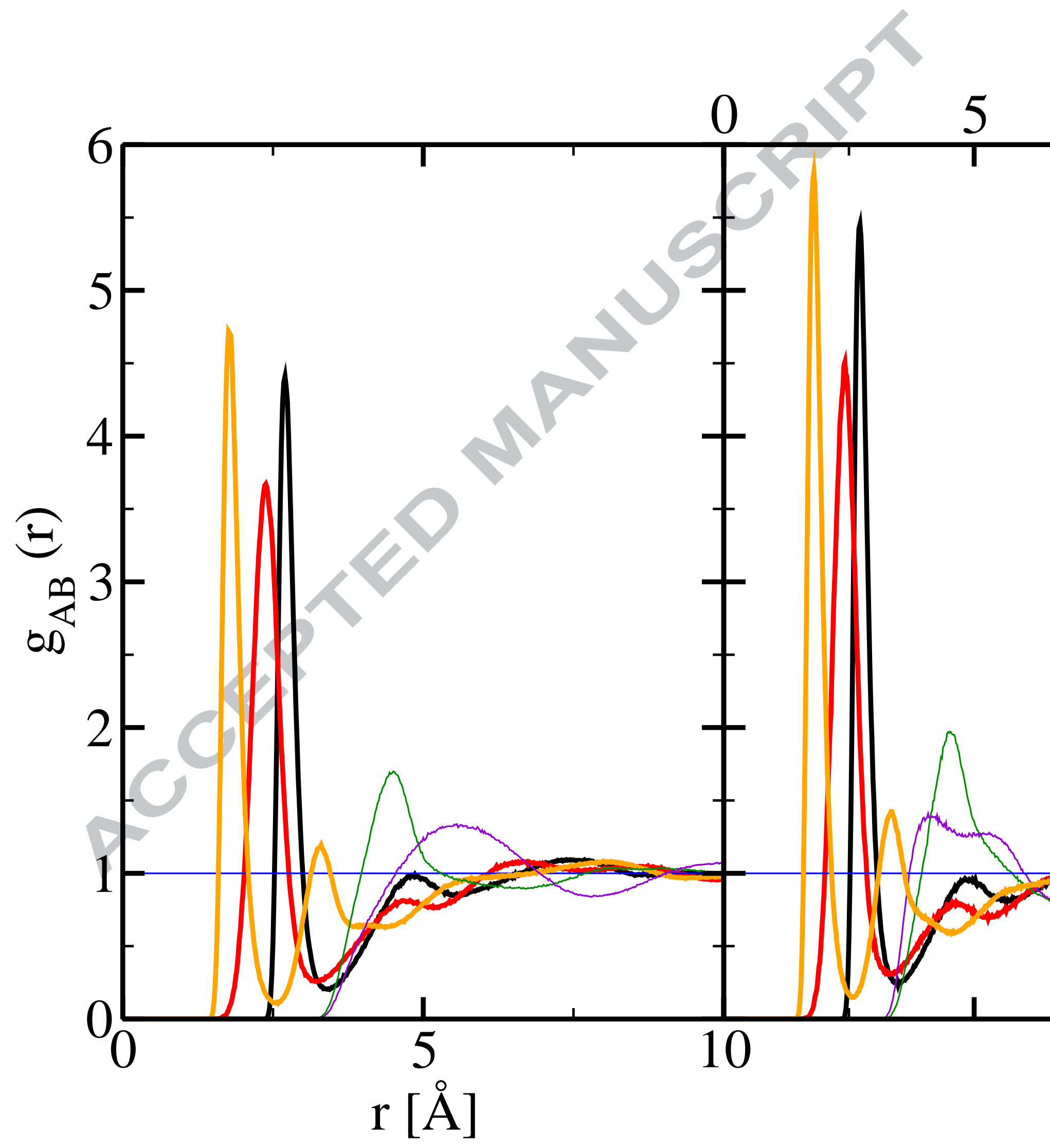

Figure 4 


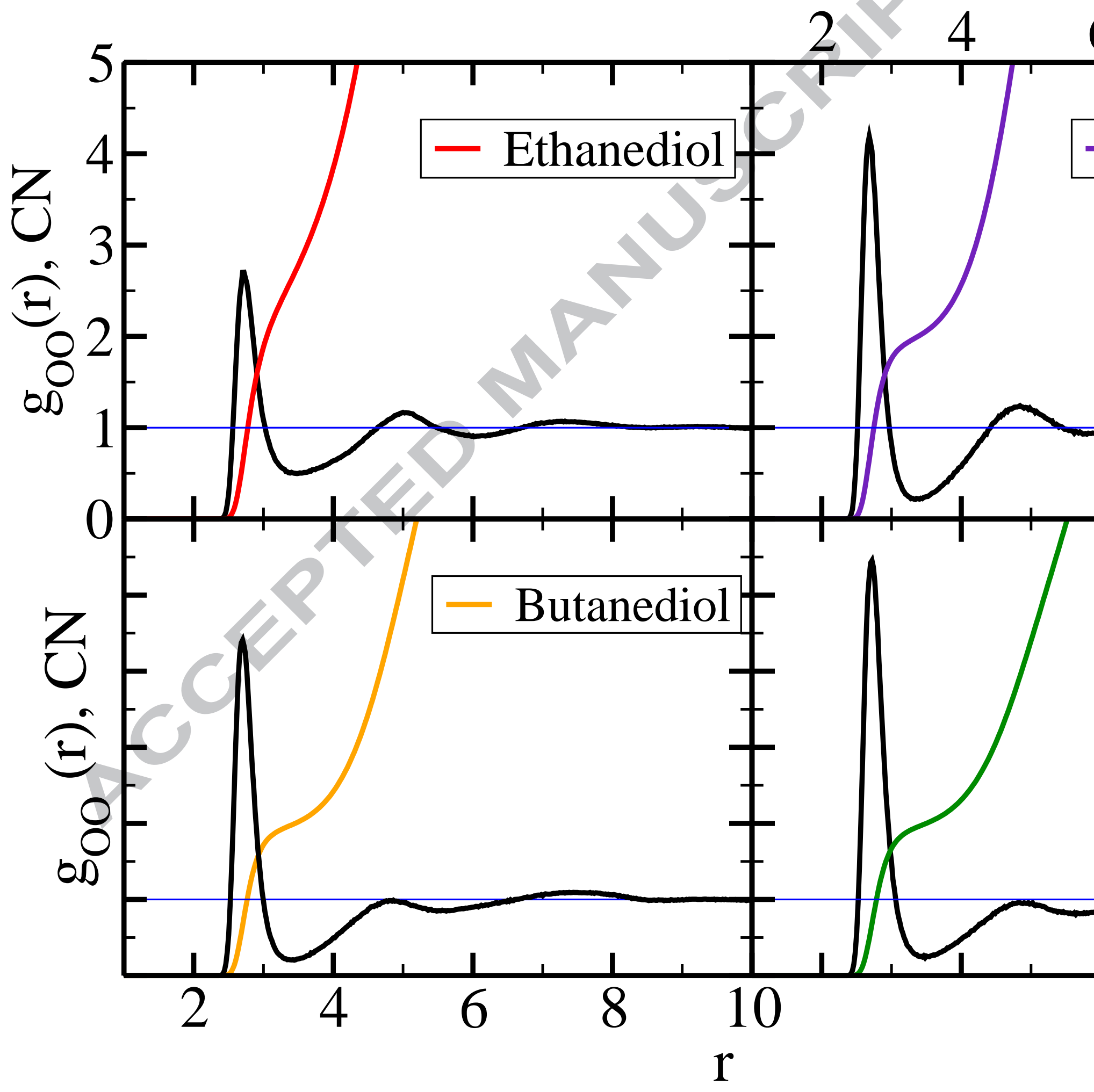


Figure 6

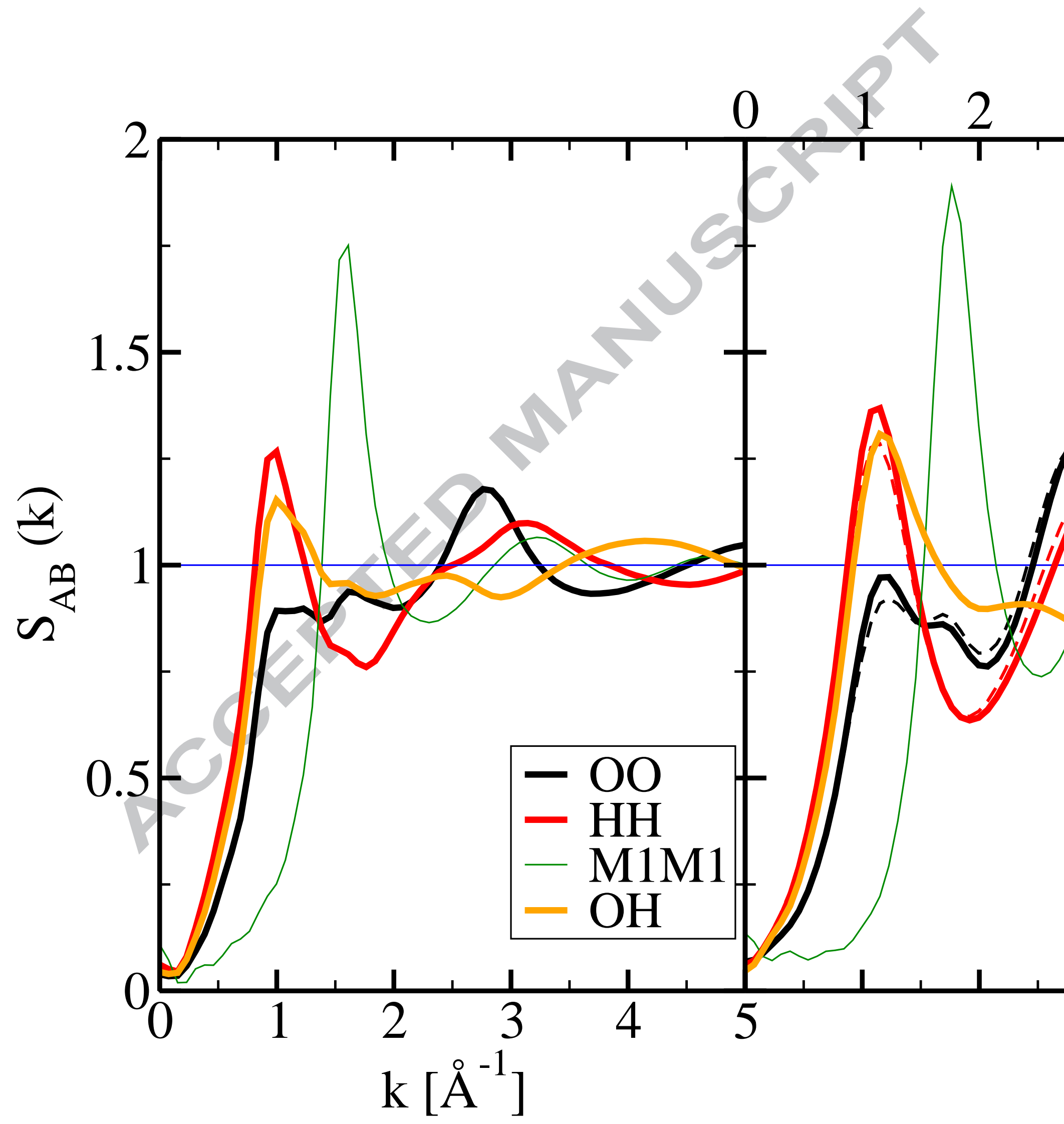




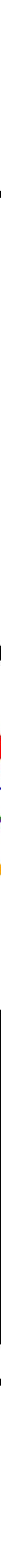

Figure 7 
Ethanediol

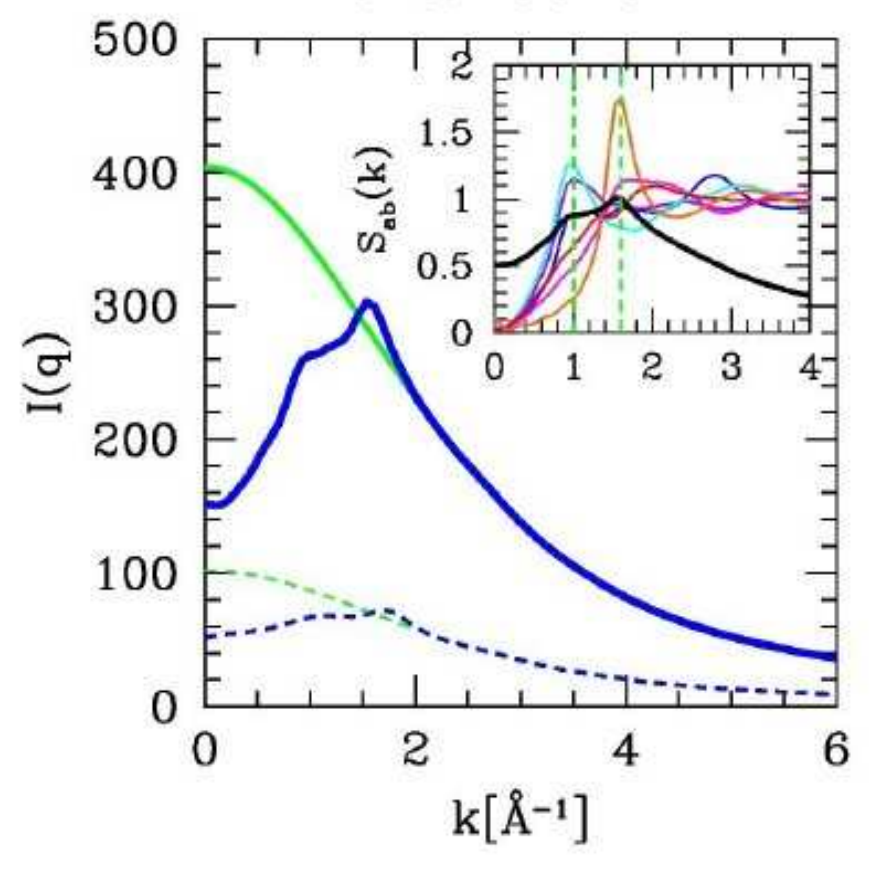

Butanediol

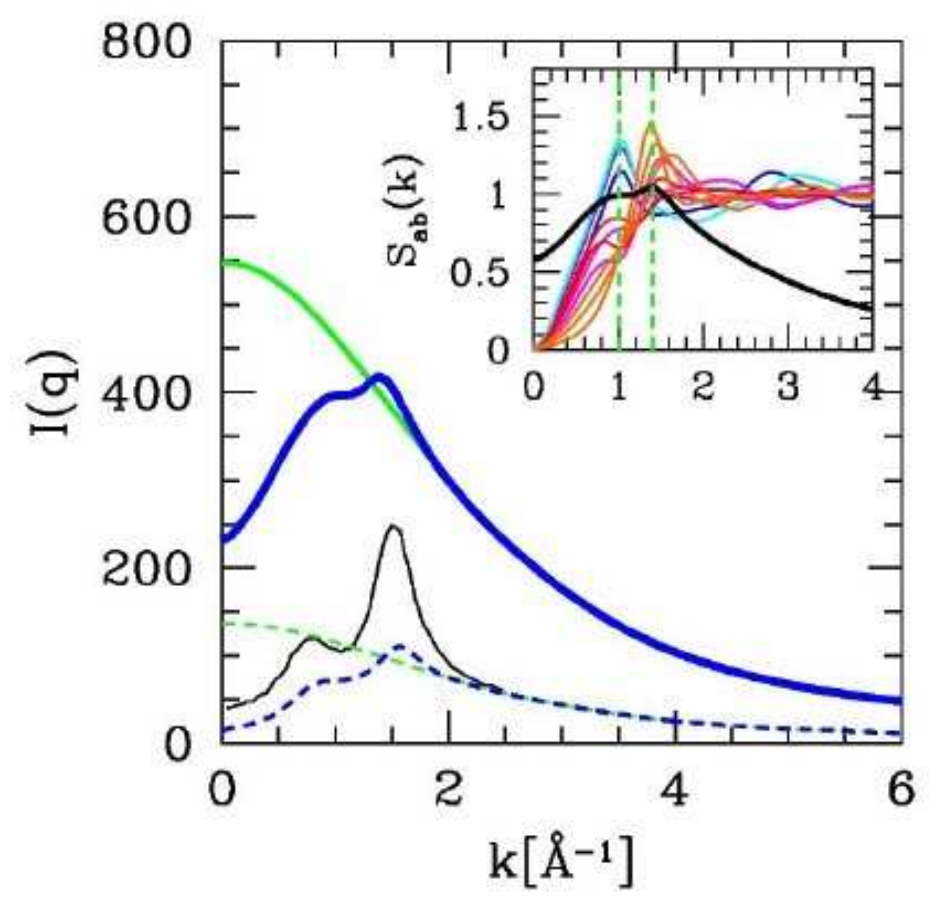

Propanediol

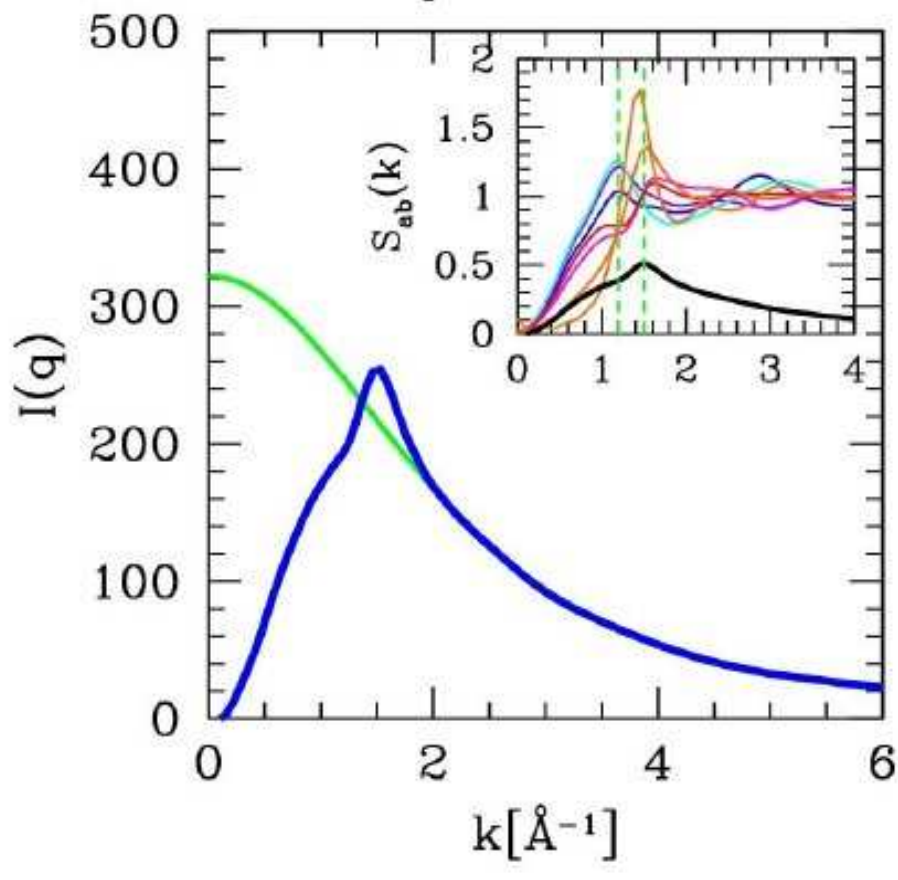

Pentanediol

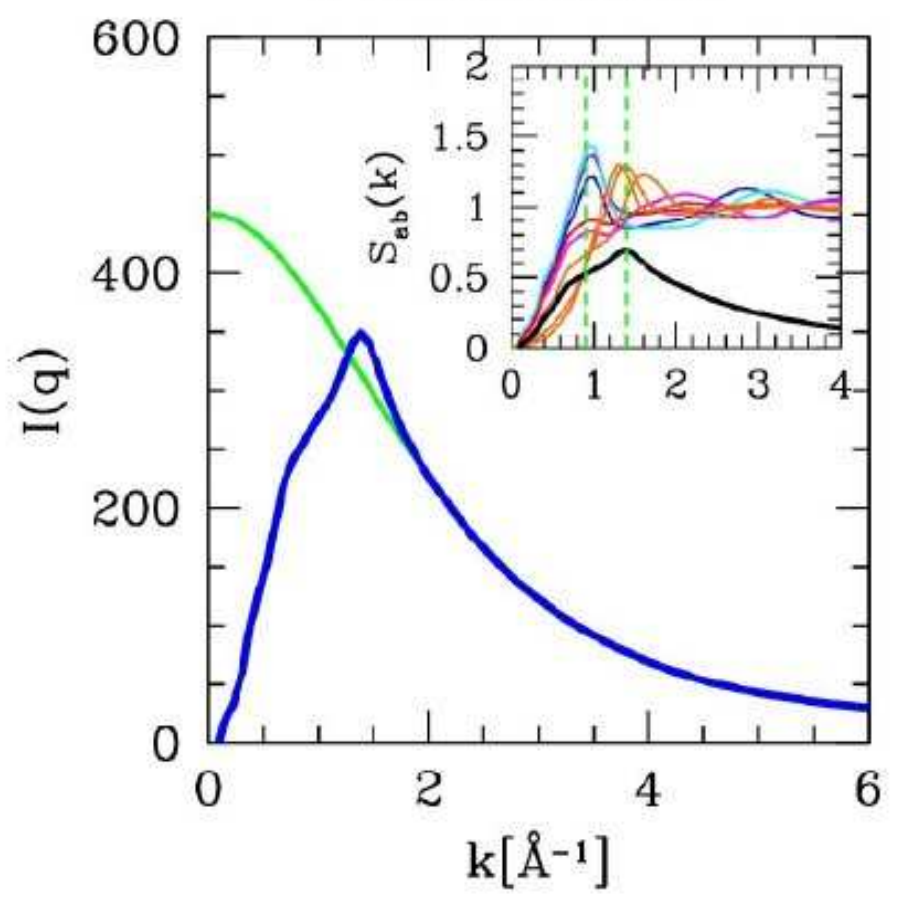


ACCEPTED MANUSCRIPT

\section{Ethanediol}

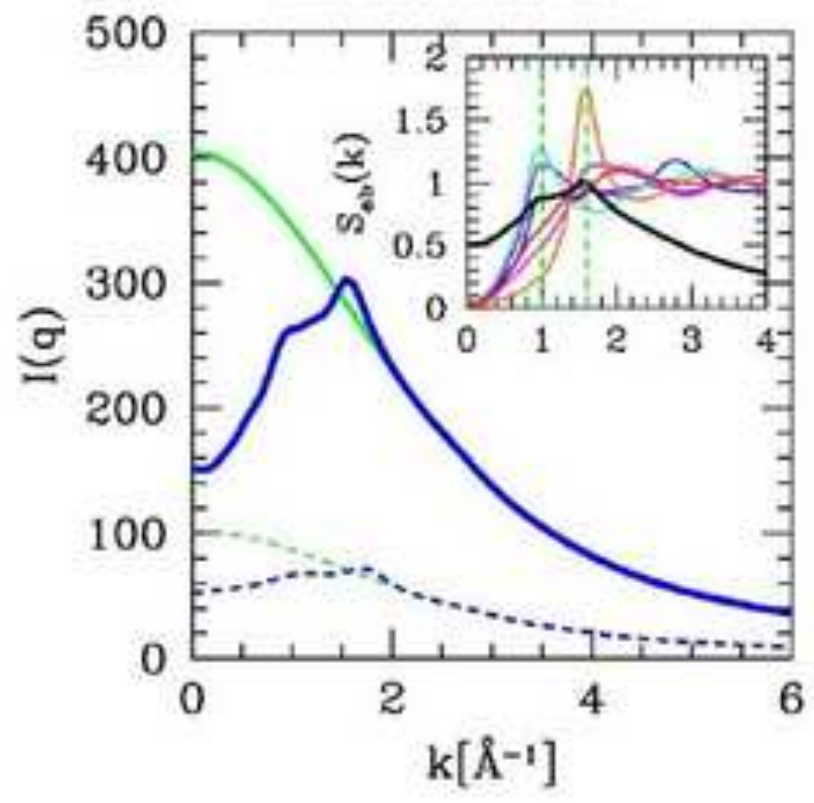

Butanediol

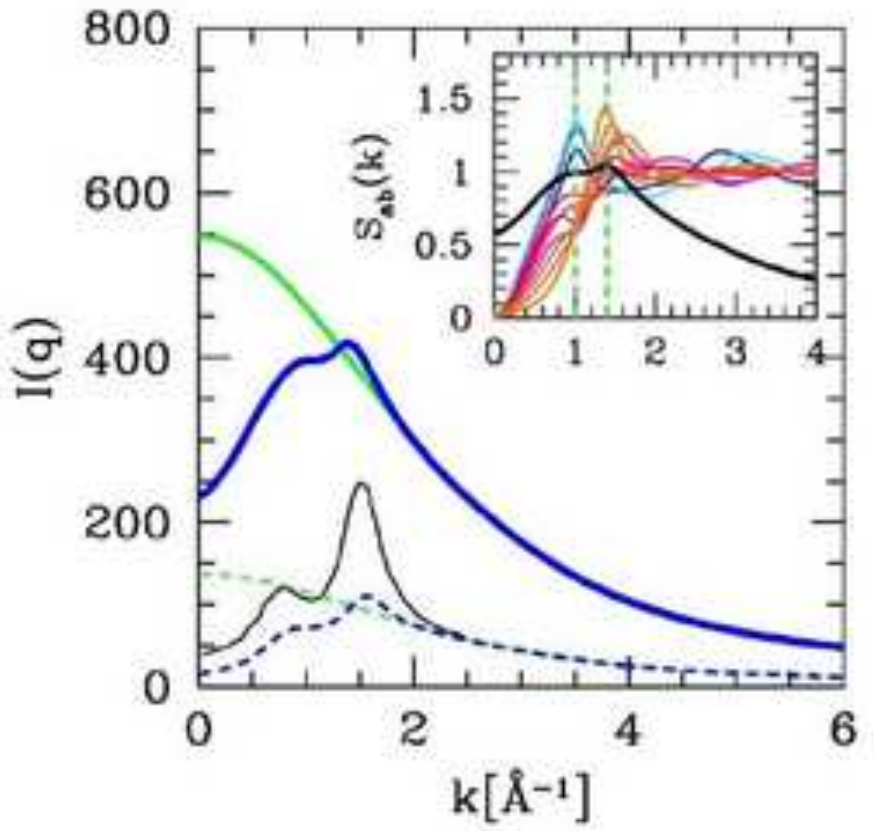

Propanediol

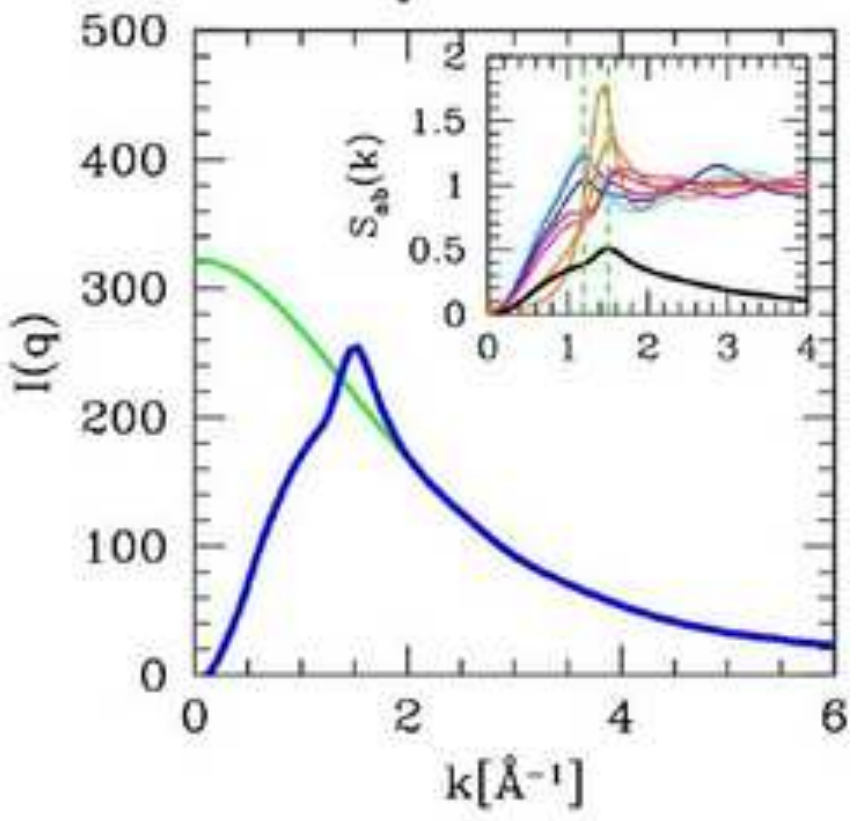

Pentanediol

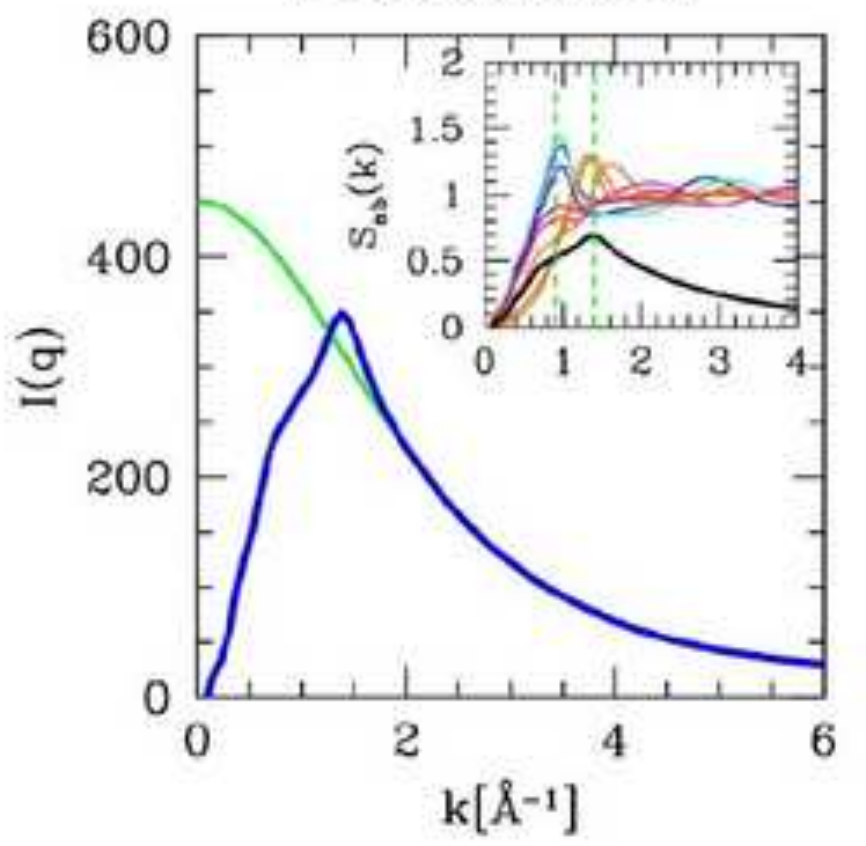


This work is about comparing the nature of self-aggregation in diols and mono-ols, both through the statistical approach (clusters, correlation functions and structure factors) and calculated Xray scattering. We find a surprising result, that the similarity in clustering between diols and mon-ols is not replicated by the similarity in the Xray scattering. The pre-peak feature known in mono-ols seems to disappear in diols. We associate this feature to the fact that hydroxyl groups of the diols are constrained by the alkyl chains. To our knowledge, this is the first report of such difference between mono-ols and diols. 